\title{
Research Mentorship Crowdsourcing Contest: Toward Global Health Good
}

\author{
Huanyu Bao, ${ }^{1,2}$ Kathryn Salisbury, ${ }^{3}$ Benjamin H. Chi, ${ }^{3}$ Joseph D. Tucker, ${ }^{1,2,3,4}$ and Weiming Tang ${ }^{1,2,5 *}$ \\ ${ }^{1}$ University of North Carolina at Chapel Hill Project-China, Guangzhou, China; ${ }^{2}$ SESH Global, Guangzhou, China; ${ }^{3}$ Institute of Global Health and \\ Infectious Disease, University of North Carolina at Chapel Hill, Chapel Hill, North Carolina; ${ }^{4}$ Faculty of Infectious and Tropical Diseases, London \\ School of Hygiene and Tropical Medicine, London, United Kingdom; ${ }^{5}$ Dermatology Hospital, Southern Medical University, Guangzhou, China
}

\begin{abstract}
As one of the six consortia funded through the NIH Fogarty International Center, the University of North Carolina at Chapel Hill, Johns Hopkins University, Morehouse School of Medicine, and Tulane University (UJMT) Fogarty Global Health Fellows Program provides postdoctoral trainees and doctoral students support and training for yearlong research attachments at selected low- and middle-income countries. To understand the current impact of this global health research training opportunity, the UJMT consortium conducted a crowdsourcing contest to gather creative messages to promote and improve the impact of the program in partnership with Social Entrepreneurship to Spur Health. Between January and March 2019, the contest received a total of 47 submissions from 14 countries; 44 were found eligible. After judging, 19 (41.2\%) submissions received a mean score of 7 or higher. We examined both textual and descriptive submissions for emergent themes and identified a range of facilitators who can be used to further improve the training programs; examples include exceptional mentorship, acquisition of research skills, career development, personal development, and multisite training opportunities. In conclusion, the crowdsourcing mentorship contest demonstrated the feasibility and acceptability of leveraging existing research networks for community engagement and how useful information can be effectively collected to highlight the effectiveness of a program and expand the reach.
\end{abstract}

\section{INTRODUCTION}

Global health research is a challenging but interesting field of study, and this attracts people with lofty ideals. The current demand for well-designed research training programs has propelled many U.S. universities to establish global health departments within their institutions. Since 2012, the Global Health Program for Fellows and Scholars, sponsored by the $\mathrm{NIH}$, has supported U.S. university consortia through collaborative, mentor-centered global health research training opportunities in low- and middle-income countries. Each consortium includes four U.S. universities, with six or more international collaborating institutes per consortium. ${ }^{1}$ As one of the six consortia, the University of North Carolina at Chapel Hill, Johns Hopkins University, Morehouse School of Medicine, and Tulane University Program (UJMT) Fogarty Global Health Fellows Program offered exceptional training for applicants at different levels of training, including postdoctoral fellows and doctoral students committed to careers in global health research. ${ }^{2}$ The program has funded more than 150 trainees in 25 countries since its inception 8 years ago.

A question that can help shed light on the impact of the program is "how has the UJMT Fogarty Global Health Fellows Program helped its fellows grow as individuals and professionals?" To expand on these discussions about the UJMT program, the UJMT consortium and the Social Entrepreneurship to Spur Health (SESH) - an organization that uses social innovation to improve health-conducted a crowdsourcing contest to gather creative messages to promote and improve the impact of the program. Crowdsourcing solicits feedback from a large and diverse group of individuals about a problem. ${ }^{3}$ Crowdsourcing contests have been widely used by governments, private foundations, and others to spur creativity-for example, the Special Programme for Research and Training in

*Address correspondence to Weiming Tang, Dermatology Hospital Southern Medical University, and University of North Carolina at Chapel Hill Project-China, No.7 Lujing Road, Guangzhou, China, 510000. E-mail: weiming_tang@med.unc.edu
Tropical Diseases (TDR) launched a crowdsourcing contest to identify creative ideas focused on increasing the number of women in a TDR mid-career clinical research fellowship. ${ }^{4}$

The implementation of the UJMT mentorship crowdsourcing contest (Figure 1) followed a practical guide published by the TDR in 2018. ${ }^{5}$ The contest included five steps: organizing a steering committee, engaging the community to contribute, evaluating the submissions, recognizing the finalists, and implementing and sharing selected ideas. First, a 12-person steering committee (including program leadership, current trainees, alumni, mentors, and organizers at SESH) was established to discuss the aim, timeline, entry types, promotion strategies, prize structures, judging criteria, and implementation plan for the contest. Second, the contest was promoted to the UJMT networks through both social media messages and a short video to reach a broad audience. The prizes, issued in the form of credit cards, were worth 1,000 U.S. dollars (USD). Third, two independent individuals screened for eligibility based on two criteria: 1) any UJMT program fellow, alumni, mentor, or in-country team members from current or former UJMT sites, and 2) the contribution should focus on how the UJMT program has helped the individual's personal and/or professional life. Fourth, three judges rated each submission on a 1-10 scale. Judging criteria were 1) how a global health experience enhanced the participants' personal or professional development and 2) participants' ideas on how to inspire the next generation of UJMT fellows. If the SD of a submission's score was greater than 2 (2), we had two new judges reevaluate it. Participants with a mean score greater than 7.0 received a commendation from the steering committee. The steering committee decided on a prize structure based on the strength of the submissions. Last, to showcase the impact and direction of the program, the UJMT consortium plans to share the most inspiring, innovative, and impressive submissions.

Between January and March 2019, the contest received a total of 47 submissions from 14 countries (Figures 2 and 3), of which 44 were eligible. After judging, 19 submissions had a 


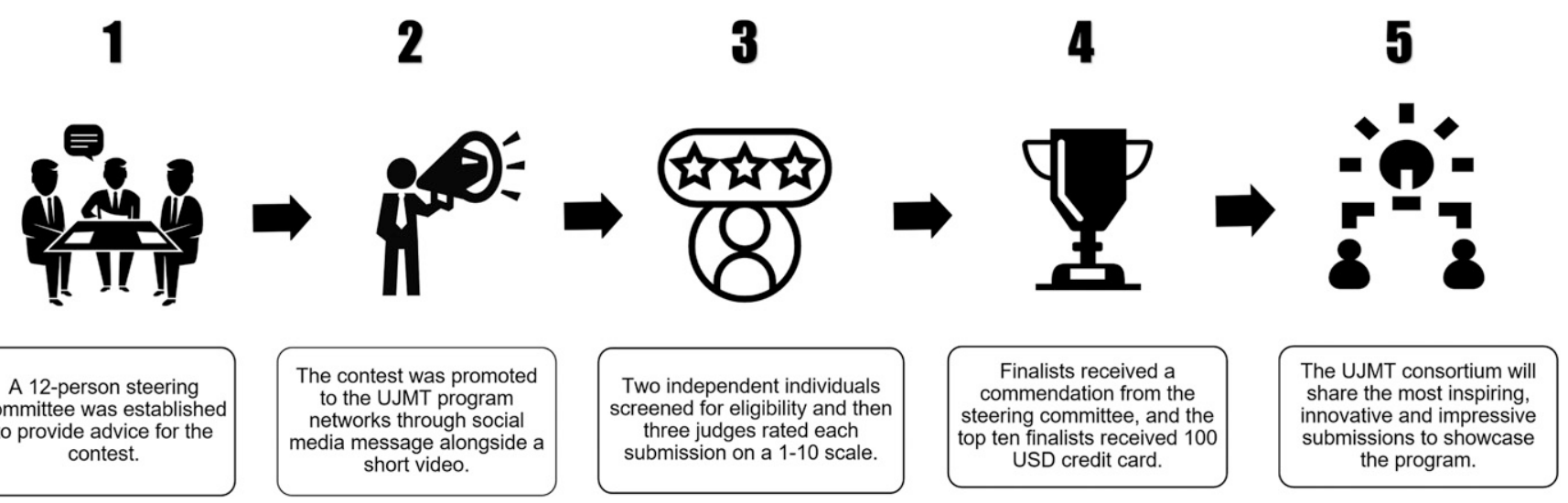

Figure 1. Overview of the University of North Carolina at Chapel Hill, Johns Hopkins University, Morehouse School of Medicine, and Tulane University mentorship crowdsourcing contest stages and function, 2019.

mean score of 7 or greater. Overall, 10 final submissions were considered exceptional, and the finalists were awarded 100 USD credit cards (see Supplement A). All finalists signed informed consent forms which included an acknowledgement that the UJMT program has permission to use the image for noncommercial purposes related to promoting the program.

All textual submissions and descriptions of image submissions were entered in Microsoft Office Excel 2016 to allow for coding (Supplement B). Two coders thematically analyzed the data using inductive and deductive coding techniques. We first used an inductive approach to openly code and identify themes related to how the program benefits both the mentors and mentees. Then, we drafted a codebook based on the results of the first round of coding. Two researchers reviewed the codebook and provided feedback. Two coders then coded the texts for a second time based on the revised codebook to deductively identify potential themes. We finalized the codebook based on the results of our second round of coding and concluded the analysis. We found five most discussed themes: 1) exceptional mentorship, 2) acquisition of research skills, 3) career development, 4) personal development, and 5) multisite training opportunities.

Exceptional mentorship. Exceptional mentorship was considered to be an essential benefit of the program. As indicated by 28 (64\%) submitted entries, the UJMT program has a strong focus on mentoring. Hands-on mentorship from experienced faculty members provides critical lessons across the entire research process, from writing proposals to manuscript preparations. For example, an alumnus reported that "Perhaps the most important aspect of the fellowship is the exposure to invaluable professional networks during the program...This step is

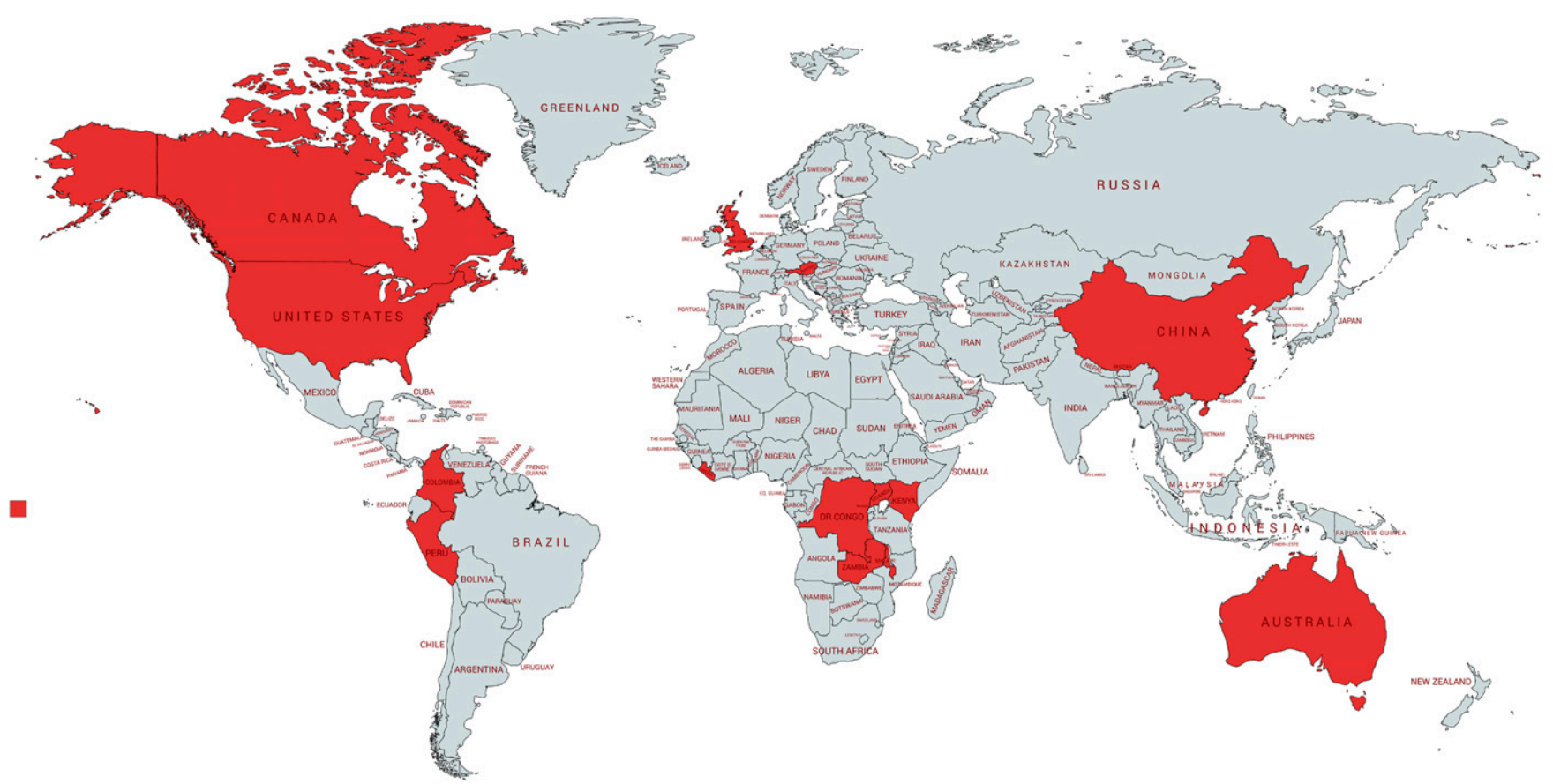

FIGURE 2. Map of countries that contributed entries to the University of North Carolina at Chapel Hill, Johns Hopkins University, Morehouse School of Medicine, and Tulane University mentorship crowdsourcing contest. This figure appears in color at www.ajtmh.org. 


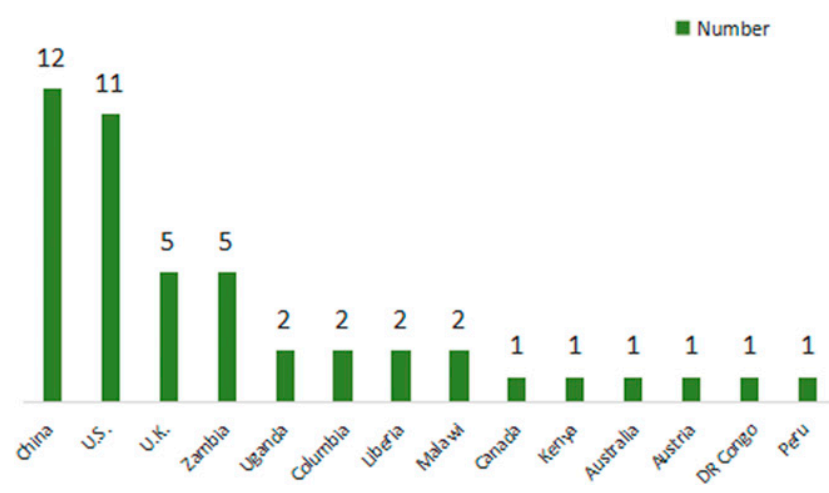

FIGURE 3. Number of entries from the countries which contributed to the the University of North Carolina at Chapel Hill, Johns Hopkins University, Morehouse School of Medicine, and Tulane University mentorship crowdsourcing contest. This figure appears in color at www.ajtmh.org.

the only step that no matter what amount of funding you receive, good mentors are primordial for the career development and engage in challenging, varied, and interesting work field."

Acquisition of research skills. Another advantage of the program commonly mentioned was the research skills fellows acquired during the program. In our analysis, 33 (75\%) entries described how the UJMT program provided mentees with the platform and guidance to develop the research skills needed to manage data collection for complex study designs. Furthermore, they improved their skills in writing research proposals and securing independent grants. For one alumnus (2015-2016), the training she received from the UJMT fellowship paved the way for her to continue research and has contributed significantly to turning her into an independent African scientist.

Career development. Career development was another recurring theme among the submissions. Several alumni reported that participation in the UJMT program confirmed their career trajectory toward global health and inspired them to pursue a career as global health researchers. For instance, through the UJMT program, a medical student (2016-2017) realized the empowering effect of global health research in alleviating health disparities and decided to pursue an academic research career on global health.

Personal development. Personal development (i.e., learning new things, becoming more confident, and broadening perspective) was also frequently emphasized. In the program, junior researchers not only developed close professional relationships but also integrated themselves into the local life and different cultures. For example, nine (20\%) participants mentioned that they learned the local language, met lifelong friends, and fell in love with the local culture. In terms of increased confidence, seven (16\%) entries showed that the experience in research, training, and mentorship enabled trainees to become confident with their research. As a current fellow (2018-2019) mentioned, "The fellowship has given me leverage to engage key stakeholders on how to formalize mentoring at the institution and propose ideas on how to establish a training program focused on helping faculty develop mentoring skills required to support investigators involved in global health research."
Multisite training opportunities. Multisite training opportunities was the last repeated theme. The UJMT program has provided ample study opportunities and built collaborative networks across the world, enabling the mentees to work on the research projects correspondent with their interests. As one trainee (2013-2014) indicated, "The program enabled me to work in Liberia, where I served as the International Medical Corps Ebola Rapid Response Initiative, leading a diverse team in implementing a helicopterbased domestic air transport system for suspected Ebola cases and infected biospecimens during the 2014-2015 West African epidemic; in Kenya, where I serve on the WHOled Malaria Vaccine Implementation Program with CDC; and Brazil, where I lead an Amazon Malaria Initiative-funded study to evaluate the efficacy of three regimens of chloroquine and primaquine to prevent relapse following Plasmodium vivax infection."

\section{DISCUSSION}

Although there are many options, crowdsourcing contest presents an innovative approach to engage members from the UJMT network to share their thoughts about the program in a short time without investing costly and time-consuming resources. This contest's patterns of online engagement were similar to health campaigns that use social media. The online approach strategy provided room for the participants to include more information, attitudes, and understanding of the subject and also allowed organizers to better assess the participants' true feelings on the program. This contest identified a range of facilitators who can be used to improve the training program. The UJMT consortium will share the most inspiring, innovative, and impressive submissions to showcase the program, and use online media tools to promote the program and attract more people to participate in global health research.

Received December 19, 2019. Accepted for publication June 2, 2020.

Published online July 20, 2020.

Note: Supplemental materials appear at www.ajtmh.org.

Acknowledgments: This study was funded by the Fogarty International Center (D43 TW009340), with additional investigator support from the National Institute of Allergy and Infectious Diseases (K24 Al120796), NIMH (R34MH119963), National Science and Technology Major Project (2018ZX10101-001-001-003), and the Nature Science Foundation of China (81903371). We thank those who participated in our contest and contributed their ideas. We also thank Emmanuela Oppong, Guangquan Shen, the steering committee members, and SESH Global for their representative contributions.

Authors' addresses: Huanyu Bao, University of North Carolina at Chapel Hill Project-China, Guangzhou, China, and SESH Global, Guangzhou, China, E-mail: huanyubao@seshglobal.org. Kathryn Salisbury and Benjamin H. Chi, Institute of Global Health and Infectious Disease, University of North Carolina at Chapel Hill, Chapel Hill, NC, E-mails: kathryn_salisbury@med.unc.edu and benjamin_ chi@med.unc.edu. Joseph D. Tucker, SESH Global, Guangzhou, China, Dermatology Hospital, Southern Medical University Guangzhou, China, Institute of Global Health and Infectious Disease, University of North Carolina at Chapel Hill, Chapel Hill, NC, and Faculty of Infectious and Tropical Diseases, London School of Hygiene and Tropical Medicine, London, United Kingdom, E-mail: jdtucker@ med.unc.edu. Weiming Tang, University of North Carolina at Chapel 
Hill Project-China, Guangzhou, China, SESH Global, Guangzhou, China, and Dermatology Hospital, Southern Medical University Guangzhou, China, E-mail: weiming_tang@med.unc.edu.

\section{REFERENCES}

1. Zunt JR et al., 2016. The National Institutes of Health Fogarty International Center global health scholars and fellows program: collaborating across five consortia to strengthen research training. Am J Trop Med Hyg 95: 728-734.
2. UNC IGHID, 2019. UJMT Fogarty Global Health Fellowship. Available at: https://globalhealth.unc.edu/education/ujmt-fogarty-globalhealth-fellowship/. Accessed March 20, 2020.

3. Tucker JD, Day S, Tang W, Bayus B, 2019. Crowdsourcing in medical research: concepts and applications. PeerJ 7: e6762.

4. Liu E et al., 2019. Women's global health leadership in LMICs. Lancet Glob Health 7: e1172-e1173.

5. World Health Organization, UNICEF, 2018. Crowdsourcing in Health and Health Research: A Practical Guide. Geneva, Switzerland: WHO. 